\title{
Psychosocial and sexual concerns of patients with implantable left ventricular assist devices: A pilot study
}

\author{
Louis E. Samuels, MD \\ Elena C. Holmes, CRNP ${ }^{a}$ \\ Ralph Petrucci, $\mathrm{PhD}^{\mathrm{b}}$
}

From the Department of Cardiothoracic Surgery, ${ }^{\mathrm{a}}$ Lankenau Hospital, Wynnewood, $\mathrm{Pa}$, and the Department of Medicine, ${ }^{\mathrm{b}} \mathrm{Di}-$ vision of Psychiatry, Hahnemann University Hospital, Drexel University, Philadelphia, Pa.

Received for publication Aug 7, 2003; revisions requested Oct 22, 2003; accepted for publication Dec 1, 2003.

Address for reprints: Louis E. Samuels, MD, Lankenau Hospital, MSB Suite 280, 100 Lancaster Ave, Wynnewood, PA 19096 (E-mail: SamuelsLE@aol.com).

J Thorac Cardiovasc Surg 2004;127:1432-5

$0022-5223 / \$ 30.00$

Copyright $\odot 2004$ by The American Association for Thoracic Surgery

doi:10.1016/j.jtcvs.2003.12.009
Objective: We sought to determine the psychosocial and sexual concerns of patients discharged from the hospital with implantable left ventricular assist devices.

Methods: Bridge-to-transplant patients with the Heartmate left ventricular assist device received a psychosocial and sexual survey at 1 month after discharge from the hospital. The survey consisted of 3 parts, with 5 questions in each category. The patients were asked to complete the survey by circling the responses and to provide more detailed answers when necessary.

Results: There were 8 male patients who completed the questionnaire. Psychologically, all patients expressed a positive mood and found support from family or religious sources. The majority described a change in attitude and behavior. Socially, all patients described a change in lifestyle, as well as a change in the reaction of family and friends. No patient went back to work or had the desire to return to work until after transplantation. The minority of patients admitted to smoking or drinking. Sexually, the majority had the desire or participated in sexual activities. The majority of patients either used or were interested in using sexual stimulants. There were no mechanical problems with the left ventricular assist devices.

Conclusions: Psychosocial and sexual conditions are altered in patients with heart failure who are discharged with implantable left ventricular assist devices. At 1 month's time interval, there is an improvement in mood, an adjustment in lifestyle, and a positive shift in relations with family and friends. There is sexual desire, with interest in sexual-enhancing medication. The most common concern is related to the pump, such as the durability of the device or damage to the components.

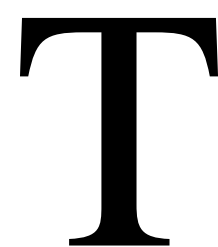

he progress in circulatory assist technology has evolved to levels that were considered unimaginable a decade ago. Advances in the field of ventricular assist device (VAD) and total artificial heart engineering have allowed surgeons to consider mechanical support as a means of permanent therapy. ${ }^{1,2}$ The ability to discharge patients from the hospital with implantable left ventricular assist device (LVAD) systems has become a standard practice, with the realization that long-term support is subject to device-related and nondevice-related issues. Although considerable attention has focused on mechanical concerns of the pumps, ${ }^{3}$ less information is known about the personal adjustment of the patients. ${ }^{4}$ The purpose of this report is to describe the psychosocial and sexual concerns of patients discharged with implantable LVADs awaiting transplantation.

\section{Patients and Methods}

Between July 1999 and March 2003, 24 patients received Heartmate implantable LVADs (Thoratec Laboratories, Inc, Pleasonton, Calif) as a bridge to transplantation. The decision for LVAD implantation was failure of maximal medical therapy to maintain stable hemodynamics or end-organ perfusion. After LVAD placement, the patients and their spouses or significant others were trained for outpatient management by the VAD nurse practitioner. 
After satisfactory training, the patients were discharged to home with weekly follow-up for 1 month, biweekly follow-up for the second month, and monthly follow-up thereafter. Only those patients who were discharged to home were included in the study. Only bridge-to-transplant patients were surveyed.

An outpatient psychosocial and sexual survey was conducted after the first month after discharge to home. This questionnaire consisted of 3 parts: psychologic, social, and sexual. Each of the 3 categories contained 5 questions that addressed mental and physical issues of the patients with respect to the presence of the pump (Table 1). This report documents the responses to the questionnaire (Table 2). The patients were asked to complete the survey with their spouse or significant other. The survey allowed for patients to qualify their answers (with an area to write in additional responses) to clarify and gain a more thorough appreciation of the responses.

\section{Results}

Eight patients were discharged from the hospital. All were men with a mean age of 50.5 years (range, 22-59 years) and a median age of 54.5 years. Five of the 8 patients were married with children, one was engaged to be married with children, and 2 patients were engaged to be married without children. All patients completed the survey. Four patients were given a diagnosis of ischemic dilated cardiomyopathy, 3 were given a diagnosis of idiopathic dilated cardiomyopathy, and one was given a diagnosis of familial cardiomyopathy.

Psychologically, all patients expressed a positive mood at the 1-month time interval. In addition, all patients found family support, religious support, or both to be helpful in their recovery. Half of the patients required psychologic counseling with the need for medication to cope with anxiety, depression, or both. The most common concerns were related to the LVADs and to their families. Specifically, the LVAD issues clustered around function and durability: Would the LVAD maintain support until heart transplantation was achieved? Family concerns were related to personal survival to be with family members, as well as family welfare from emotional and financial aspects. Socially, all patients reported a change in their lifestyle to accommodate the LVAD. There were positive changes noted, such as increased activity level, endurance, and overall energy levels. There were alterations in daily living related to the LVAD, such as driveline site maintenance, washing, battery charging, and transportation limitations. The interactions with family and friends were changed because of the LVAD. A deeper concern and a closer relationship with people were observed on all accounts. On occasion, a celebrity-type status was described by several individuals. Although none of the patients worked, half expressed an interest in returning to work after recovery from transplantation. Three of the 8 patients admitted to drinking alcoholic beverages or smoking: these activities were irregular and infrequent.
TABLE 1. Psychosocial and sexual survey (circle response, write in additional thoughts)

Psychological

1. Has your emotional state changed since the LVAD? Yes, No, Other

2. What are your top three concerns? Myself, The LVAD, Other

3. Have you received psychological counseling or medication? Yes, No

4. Has your attitude and behavior changed since receiving the LVAD? Yes, No

5. Do you find religion and/or support groups/services to be helpful? Yes, No, Other

Social

1. Have you had occasion to smoke, drink (alcohol), or partake in recreational drug use? Smoke, Alcohol, Drugs, Other

2. Do you socialize and interact more or less with friends and family since the LVAD? More, Less, Same

3. Have you gone back to work or desire to do so? Yes, No, Other

4. Has your lifestyle changed since the LVAD? Yes, No, Other

5. Are your family and friends reacting to your situation any differently? Yes, No, Other

Sexual

1. Do you have the desire and/or have you engaged in sexual activity? Yes, No, Other

2. What are your concerns about sexual activity on the LVAD? Myself, The LVAD, My partner, Other

3. Have you experienced any problems with your performance or the LVAD's function during sexual activity? Yes, No, Other

4. Have you had the need for any medications, such as Viagra? Medication, No Medication, Other

5. Has your attitude about sex changed since the LVAD surgery? Yes, No, Other

LVAD, Left ventricular assist device.

Sexually, 7 of the 8 patients were sexually interested (n $=2)$ or sexually active $(\mathrm{n}=5)$ within 1 month after discharge. Although there were no problems encountered with the LVAD during sexual activities, patients did express concern about the device, particularly the driveline. Interestingly, one patient was concerned about the ability to have children, thinking the presence of the machine made him sterile. The attitude toward sexual behavior was mixed, with half the patients describing a change in a positive direction. Correspondingly, the majority of patients were interested (no. 3) in or actually used (no. 3) sexual stimulants. No adverse events were reported in those patients who used sildanafil or alprostadil.

\section{Discussion}

The psychosocial and sexual issues of patients with implantable LVADs are becoming a visible part of the overall care of patients using these technologies. It is a stark realization that patients discharged with LVADs are engaging in activ- 
TABLE 2. Responses (abbreviated) to psychosocial and sexual survey

\begin{tabular}{|c|c|c|c|c|c|c|c|c|}
\hline & \multicolumn{8}{|c|}{ Patient no. } \\
\hline & 1 & 2 & 3 & 4 & 5 & 6 & 7 & 8 \\
\hline Age (y) & 54 & 57 & 22 & 50 & 57 & 59 & 50 & 55 \\
\hline \multicolumn{9}{|l|}{ Psychologic } \\
\hline Positive mood & Yes & Yes & Yes & Yes & Yes & Yes & Yes & Yes \\
\hline Concerns & VAD & VAD & VAD & VAD & Family & VAD & Family & VAD \\
\hline Counseling & No & Yes & No & Yes & Yes & No & No & Yes \\
\hline Behavior change & No & Yes & Yes & Yes & Yes & No & Yes & Yes \\
\hline \multirow[t]{2}{*}{ support } & Yes & Yes & Yes & Yes & Yes & Yes & Yes & Yes \\
\hline & $(\mathrm{F})$ & $(\mathrm{R} / \mathrm{F})$ & $(\mathrm{R} / \mathrm{F})$ & $(\mathrm{R} / \mathrm{F})$ & $(\mathrm{F})$ & (R/F) & (R/F) & $(\mathrm{F})$ \\
\hline \multicolumn{9}{|l|}{ Social } \\
\hline Substances & No & Smoke & $\mathrm{EtOH}$ & No & No & No & $\mathrm{EtOH}$ & No \\
\hline Socialize & Same & Same & More & Same & Same & Same & More & Same \\
\hline Work & No & No & No & No & No & No & No & No \\
\hline Lifestyle change & Yes & Yes & Yes & Yes & Yes & Yes & Yes & Yes \\
\hline $\begin{array}{l}\text { Family/friends change in } \\
\text { relations }\end{array}$ & Yes & Yes & Yes & Yes & Yes & Yes & Yes & Yes \\
\hline \multicolumn{9}{|l|}{ Sexual } \\
\hline Active & No & Desire & Yes & Yes & Desire & Yes & Yes & Yes \\
\hline Concerns & None & Fatigue & Children & LVAD & ED & None & LVAD & LVAD \\
\hline Problems & NA & NA & None & None & $\mathrm{ED}$ & None & None & None \\
\hline Medications & No & Viagra & Viagra & MUSE & Viagra & Viagra & Viagra & No \\
\hline Attitude change & No & Yes & Yes & Yes & No & Yes & No & No \\
\hline
\end{tabular}

$V A D$, Ventricular assist device; $F$, family; $R$, religion; $E t O H$, alcohol; $L V A D$, left ventricular assist device; $E D$, erectile dysfunction; $N A$, Not applicable; Viagra, sildanafil; MUSE, alprostadil.

ities well beyond those covered in the package insert. Conversations with VAD practitioners from numerous VAD centers are filled with stories of outpatients involved with functions that were inconceivable a decade ago. Patients are playing sports, attending social events, and traveling worldwide. The limits of activities are measured only by the imagination. Indeed, the preparation and management of patients with LVADs discharged from the hospital has become a specialty of the VAD practitioner. ${ }^{5}$

Although the majority of our attention has focused on the mechanical aspects of the technology and the medical condition of the patient, a far less obvious need resides in the emotional and mental well being of the LVAD recipient. Despite the engineering aspects of the pump (ie, the durability and functionality of the system), the systemic effects of artificial technology in the human species weighs heavily on the psyche. The overall concern of patients with LVADs was addressed in the question that asked what the biggest concern was while receiving support. The answers clustered around 2 responses: the durability of the pump and the duration of pump dependence before a transplant. These patients were clearly worried about the device breaking down over time and the uncertainty of when a donor organ would become available. An analogous scenario was observed in patients with other implanted cardiac devices, such as pacemakers and defibrillators. For some, the implanted device was readily accepted. For others, it was a symbol of loss, debilitation, independence, and resilience. ${ }^{6}$
Regarding the findings of this survey with respect to specific psychosocial and sexual concerns, it is clear that the majority of patients had an improvement in their condition compared with baseline. Overall, patients felt better. The LVAD, by virtue of its ability to improve cardiac output, reversed many of the physical side effects of heart failure. In so doing, the patients' moods were uplifted, relations with family and friends were restored or enhanced, and sexual desires were fulfilled. Interestingly, these findings forced us to reconsider the management of patients with heart failure receiving mechanical assistance or replacement technologies. For example, based on early studies by Masters and Johnson, ${ }^{7}$ a "cultural mythology" was adopted in which sexual activity in patients with significant cardiovascular disease was not recommended. Guidelines for the management of sexual dysfunction in patients with cardiovascular disease were adopted, ${ }^{8}$ with careful consideration toward those patients with advanced degrees of heart failure (ie, class III-IV). The use of sildanafil (Viagra), for example, was contraindicated in patients with congestive heart failure. Implantation of an LVAD, however, automatically changes the profile of patients with heart failure. Limitations and restrictions in activities, sexual or otherwise, need to be reconsidered. Adjustments in medications, such as $\beta$-blockers, angiotensin-converting enzyme inhibitors, and nitrates, need to be reassessed. The use of medications that were otherwise considered contraindicated in heart failure need to be revised. 
In summary, there is a wide spectrum of concerns in patients supported with implantable LVADs. Although the number of patients is small, there are several emerging patterns. There are direct issues related to the technology, and there are concerns related to the patient's psychologic homeostasis with himself and with others. Although this patient population was small and limited to bridge-to-transplant men, the results suggest that destination therapy patients of either sex might encounter similar findings. In this regard the tracking and understanding of psychosocial and sexual data needs to be refined. Although the questionnaire used in this report gives practical insight regarding the concerns of outpatients with implantable LVADs, the survey's use as a standardized tool is limited. The Minnesota Living with Heart Failure questionnaire, on the other hand, is a well-established measure of the patients' perceptions of the effects of congestive heart failure on their lives. ${ }^{9}$ This resource, in a modified form to address patients with LVADs, might be of value. In short, this report serves as a stimulus for future study.

\section{Conclusions}

Patients discharged with implantable LVADs have psychosocial and sexual concerns that are related to the technology and its effects on the mind and body. Overall, there is an improvement in mood at 1 month's time. There are changes in lifestyle to accommodate the presence of the pump, alterations in the relationships between friends and family, and a desire toward intimacy. Recognition of these mental and emotional states is critical to our global management of patients with implantable LVADs. A standardized form needs to be created to more completely analyze these sensitive areas and others.

\section{References}

1. Kukuy EL, Oz MC, Rose EA, Naka Y. Devices as destination therapy. Cardiol Clin. 2003;21(1):67-73.

2. Samuels LE, Dowling R. Total artificial heart: destination therapy. Cardiol Clin. 2003;21(1):115-8.

3. Sun BC, Catanese KA, Spanier TB, Flannery MR, Gardocki MT, Marcus LS, et al. 100 Long-term implantable left ventricular assist devices: the Columbia Presbyterian interim experience. Ann Thorac Surg. 1999;68(2):688-94.

4. Buda TM, Kendall K. Nursing and psychosocial issues of patients on mechanical support. J Card Surg. 2001;16(3):209-21.

5. Holmes EC. Outpatient management of long-term assist devices. Cardiol Clin. 2003;21:93-9.

6. Beery T, Baas L. Medical devices and attachment: holistic healing in the age of invasive technology. Issues Ment Health Nurs. 1996;17(3):23343.

7. Strassman HD. Sex and the work of Masters and Johnson. GP. 1968; 38(4):109-14.

8. DeBusk R, Drory Y, Goldstein I, Jackson G, Kaul S, Kimmel SE, et al. Management of sexual dysfunction in patients with cardiovascular disease: recommendations of The Princeton Consensus Panel. Am J Cardiol. 2000;86(2):175-81.

9. Rector TS, Kubo SH, Cohn JN. Patients self-assessment of their congestive heart failure. Part 2: Content, reliability and validity of a new measure, the Minnesota Living with Heart Failure questionnaire. Heart Failure. 1987;2:198-209. 